\title{
Human Leucocyte Antigen B50 Is Associated with Conversion to Generalized Myasthenia Gravis in Patients with Pure Ocular Onset
}

\author{
Bedile Irem Tiftikcioglu ${ }^{a} \quad$ Irem Fatma Uludag $^{\mathrm{a}} \quad$ Yasar Zorlu $^{\mathrm{a}} \quad$ İbrahim Pirim $^{\mathrm{b}, \mathrm{c}}$ \\ Ufuk Sener $^{\mathrm{a}}$ Figen Tokucoglu ${ }^{\mathrm{a}}$ Meltem Korucuk ${ }^{\mathrm{a}}$ \\ ${ }^{a}$ Department of Neurology, and ${ }^{b}$ Tissue Typing Laboratory, Department of Genetics, TCSB Izmir Tepecik Education and \\ Research Hospital, and 'Department of Medical Biology, Katip Celebi University School of Medicine, Izmir, Turkey
}

\section{Key Words}

Myasthenia gravis - Genetic susceptibility · Human leucocyte antigen - Major histocompatibility complex . Ocular onset

\begin{abstract}
Objectives: The aim of this study was to investigate the associations between major histocompatibility complex (MHC) class I and II alleles and disease characteristics in Turkish patients with myasthenia gravis (MG). Subjects and Methods: The MHC class I and II alleles of 108 unrelated MG patients were genotyped. The human leucocyte antigen (HLA) distribution of all MG patients and subgroups of MG patients (grouped according to disease characteristics) was compared to that of 250 healthy controls. Results: Overall distributions of HLA-B*61 and $C^{*} 05$ were more frequent in MG patients (7.4 vs. $2.0 \%$ and 14.8 vs. $6.8 \%$, respectively) than in non-MG patients. Subgroup analyses revealed that HLADRB $1 * 14$ and $\mathrm{DQB} 1 * 02$ alleles were more frequent in earlyonset MG $[n=10(20.8 \%)$ vs. $\mathrm{n}=25(10.0 \%)$ and $n=21(43.8 \%)$ vs. $n=59(23.6 \%)]$. In patients seropositive for anti-AchR antibodies, the frequencies of $\mathrm{HLA}-\mathrm{B}^{*} 50$ and $\mathrm{C}^{*} 05$ were higher. HLA-C*05, DRB $1 * 01$, and DRB $1 * 11$ were higher in patients with ocular MG. In addition, HLA-A*01, A*31, B*08, and DRB1*14 were higher among patients with thymic hyperpla-
\end{abstract}

\begin{tabular}{ll}
\hline KARGER & ( 2016 S. Karger AG, Basel \\
$\begin{array}{l}\text { E-Mail karger@karger.com } \\
\text { www.karger.com/mpp }\end{array}$ & $\begin{array}{l}\text { This is an Open Access article licensed under the terms of the } \\
\text { Creative Commons Attribution-NonCommercial 3.0 Un- } \\
\text { ported license (CC BY-NC) (www.karger.com/OA-license), } \\
\text { applicable to the online version of the article only. Distribu- } \\
\text { tion permitted for non-commercial purposes only. }\end{array}$
\end{tabular}

sia, whereas DQB1*03 was lower. However, all of these differences lost significance after correction of the $p$ value for multiple comparisons. No allele association was found among patients with thymoma. Strikingly, patients with generalized MG who had pure ocular symptoms at disease onset had significantly increased HLA-B*50 compared to the controls (corrected $p<0.001, \mathrm{OR}=9.92 ; 95 \% \mathrm{Cl} 3.05-32.22$ ). Conclusion: The HLA-B*50 allele was associated with conversion to generalized disease in patients with pure ocular symptoms at disease onset. This finding could extend our understanding of the complex interactions between the pathogenesis of MG and genetic heritage.

(c) 2016 S. Karger AG, Basel

\section{Introduction}

Myasthenia gravis (MG) is an autoimmune disorder of the nervous system that affects the neuromuscular junctions, leading to muscle weakness and fatigue [1]. Antibodies against acetylcholine receptors (AChR), detected in the majority (85-90\%) of patients, are highly specific and pathogenic; however, seropositive patients still display a widely heterogeneous clinical spectrum [1]. This heterogeneity had been attributed to possible genetic and environmental factors underlying the susceptibility to

Bedile Irem Tiftikcioglu

Department of Neurology, TCSB Izmir Tepecik Education and Research Hospital Gaziler cad. No. 468, Yenisehir

TR-35170 Izmir (Turkey)

E-Mail irem.tiftikcioglu@gmail.com 
MG [2]. There is extensive data documenting the importance of genetic factors in predisposition to various autoimmune disorders $[2,3]$. In MG, initial evidence supports that the genetic predisposition accumulated from familial studies showing an increased risk of $2-4 \%$ for the relatives of the MG patients is significantly higher than the prevalence of the disease in the general population [4].

Every individual expresses a different combination of immunoregulatory genes [i.e., human leucocyte antigen (HLA) genes and many non-HLA genes], which determines their T-cell repertoire. The balance between subgroups of T-lymphocyte subsets determines the immune tolerance [5]. In most cases, the selective loss of immune tolerance to self-nicotinic AchR antigens, and the production of specific antibodies, leads to impaired signal transmission in neuromuscular junctions [6]. Several studies have shown the presence of AChR-specific autoreactive $\mathrm{T}$ cells and $\mathrm{AChR}$ antibody-producing $\mathrm{B}$ cells in MG patients $[7,8]$. Although the factors triggering the breakdown of self-tolerance have not yet been determined in MG, several environmental factors, such as viral infections and drugs, have been suggested $[9,10]$.

Human major histocompatibility complex (MHC) represents the strongest susceptibility locus for autoimmune diseases and contains HLA and many other genes that exert a major influence on most immune responses [3]. These have been extensively investigated as possible targets for predisposing or preventive genes in autoimmune diseases $[3,11]$. Since 1972, many studies have been conducted to clarify the genetic basis of MG, and several different HLA alleles have been associated with this disease in different ethnic populations [12-15]. The aims of the present study were to investigate the distribution of MHC class I (HLA-A, HLA-B, and HLA-C) and II (HLADRB1 and HLA-DQB1) alleles in a group of MG patients of Turkish ethnic origin, and to further investigate the associations between HLA alleles and different clinical subgroups of the disease.

\section{Subjects and Methods}

\section{Subjects}

Two hundred nine patients with a diagnosis of MG, who were treated in the Neuromuscular Diseases Unit of our tertiary-care medical center between 2000 and 2013, were screened. The inclusion criteria were: diagnosis of acquired autoimmune MG and Caucasian Turkish origin as assessed by the ethnicity of 4 generations. The exclusion criteria were: family relationship, lack of regular follow-up visits, and death. Of these 209 patients, 124 fulfilled the study criteria, and of these, 108 patients (61 females) between the ages of 22 and 90 years agreed to participate in this study. All of the patients included in this study were from Western Anatolia. The control group consisted of 250 randomly selected, healthy, unrelated individuals with ethnic backgrounds similar to those of the patients, who were registered as blood donors at the Blood and Tissue Bank of Izmir Tepecik Education and Research Hospital. This study was approved by the local ethics committee and written informed consent was obtained from all of the study participants.

The diagnosis of acquired autoimmune MG was made according to clinical, immunological, and electrophysiological diagnostic criteria, as well as a positive neostigmine test when required. The demographic and clinical data were obtained from medical records. Clinical features, such as age at disease onset, initial symptoms at onset, seropositivity for anti-AchR antibodies, clinical disease type, and thymus histopathology, were noted. Since 96 of the 108 patients had not been previously tested for MG-related autoantibodies such as anti-MuSK, anti-titin, and anti-LRP4, only the results of anti-AchR antibody testing were taken into consideration as a serological marker in this study. Patients with disease onset before the age of 40 years were considered to have earlyonset MG (EOMG). Patients between the ages of 40 and 65 years were considered to have late-onset MG (LOMG), and patients older than 65 years were grouped as having very-late onset MG. The initial symptoms at disease onset were classified as ocular weakness, bulbar weakness, or limb weakness. Clinical disease types were determined according to the disease distribution after at least 2 years of follow-up (ocular vs. generalized). Forty-nine patients had previously undergone thymectomy. Thymic pathology was defined as follows: thymic hyperplasia was the appearance of lymphoid follicles not ordinarily seen in the normal adult thymus, while thymic atrophy was the presence of fatty replacement of thymic tissue. Thymoma was defined by the presence of a tumor containing thymus-specific epithelial cells together with a varying number of lymphocytes. Thymus hyperplasia was the most frequent pathological finding among all of the patients with thymectomy in the study group.

First, the HLA allele distribution of the entire patient group was compared to that of the controls. The MG patients were then subgrouped according to different disease characteristics, such as age at the onset of disease, seropositivity for anti-AchR antibodies, having only ocular findings at onset, clinical disease type, thymic pathology, and concomitant autoimmune disease. The MG patients' allele distribution was then compared with that of the control group to further assess HLA relationships in more homogeneous patient populations. Finally, the HLA allele associations in male and female MG patients were investigated, separately.

\section{Deoxyribonucleic Acid Extraction and Genotyping}

Peripheral venous blood samples $(3 \mathrm{~mL})$ were collected from the patients and control subjects into test tubes containing ethylenediamine tetraacetic acid. Genomic deoxyribonucleic acid (DNA) samples were extracted from whole blood using Genovision GenoM-6 (GenoVision Inc., West Chester, PA, USA), which is a magnetic bead-based, fully automated DNA isolation and purification system, according to the manufacturer's instructions. Low-resolution HLA typing was performed by polymerase chain reaction using sequence-specific oligonucleotide probe assays (Lifecodes HLA SSO Typing Kits; Gen-Probe Transplant Diagnostics Inc., Stanford, CT, USA) and a Luminex 200 analyzer (Luminex Corp., TX, USA) with Luminex IS 2.3 software. 
Statistical Analysis

Statistical analysis was performed using SPSS for Windows v.15.0 (SPSS Inc., Chicago, IL, USA). The demographical and clinical data of MG patients are presented using frequency analysis. The presence or absence of alleles in the patient and control groups was compared using $2 \times 2$ contingency table analysis, calculations were done using Pearson's $\chi^{2}$ tests, and Fisher's exact test was used when any of the expected frequencies was less than 5 . Associations were corrected for multiple comparisons using Bonferroni's correction [i.e., the significance value $(p)$ was multiplied by the number of variables tested, e.g., 78 class I and II HLA alleles detected, so $P c=P \times 78$ ]. $p<0.05$ was considered statistically significant.

\section{Results}

The clinical features of the MG patients are summarized in Table 1. The median age of the patients at disease onset was 43.0 years. Eighty-one $(81 ; 75 \%)$ patients with MG were seropositive for anti-AchR antibodies. The rate was $61.9 \%(n=13)$ in patients with ocular MG, $82.1 \%$ $(n=23)$ in patients with thymic hyperplasia, and $91.7 \%$ $(n=11)$ in patients with thymoma.

Ocular weakness was the most common initial presentation of MG, occurring in 79 (73.1\%) patients. Among the initial symptoms, limb weakness was present in 44 (40.7\%) patients, while bulbar weakness was present in 28 (25.9 \%) patients. Forty-eight $(48,44.4 \%)$ MG patients had pure ocular weakness at disease onset; however, 27 (56.3\%) of these cases progressed to generalized weakness over time. Based on the clinical disease type, 21 (19.4\%) patients were diagnosed with ocular MG on follow-up.

Typing of the HLA-A, HLA-B, HLA-C, HLA-DRB1, and HLA-DQB1 alleles was performed for all cases. The relative prevalence of individual MHC class I and II alleles in the MG patients and controls is summarized in Tables 2 and 3, respectively. The overall distribution of HLA alleles $B^{*} 61$ and $C^{*} 05$ was more frequent in MG patients (Table 2); however, these differences lost significance after correction of the $p$ values $(P c=1.248$ and $P c=0.936$, respectively).

Subgroup analyses according to different disease characteristics revealed several allele associations in the MG patients compared to the controls. The significant data found in the analysis of the MG patient subgroups are summarized in Table 4; however, these differences lost significance after correction for multiple comparisons. There was no significant HLA allele association with the disease in relation to gender.

Interestingly, the patients with generalized MG who had pure ocular symptoms at disease onset $(n=27)$ had significantly higher HLA-B*50 $(\mathrm{n}=6,22.2 \%)$ compared
Table 1. Demographic and clinical data of the MG patients

\begin{tabular}{|c|c|c|}
\hline Feature & Patients & $\begin{array}{l}\text { Female/male } \\
\text { ratio }\end{array}$ \\
\hline Age, years ${ }^{\mathrm{a}}$ & $53.7 \pm 16.9$ & \\
\hline Age at disease onset, years ${ }^{\mathrm{a}}$ & $43.9 \pm 18.5$ & \\
\hline \multicolumn{3}{|l|}{ Age at disease onset } \\
\hline$<40$ years (early onset) & $48(44.4)$ & $36 / 12$ \\
\hline$\geq 40$ years (late onset) & $60(55.6)$ & $25 / 35$ \\
\hline \multicolumn{3}{|l|}{ Anti-AchR antibody status } \\
\hline Negative $(<0.4 \mathrm{nM})$ & $27(25.0)$ & $17 / 10$ \\
\hline Positive $(\geq 0.4 \mathrm{nM})$ & $81(75.0)$ & $44 / 37$ \\
\hline \multicolumn{3}{|c|}{ Presenting symptoms at disease onset } \\
\hline Ocular weakness & $79(73.1)$ & $42 / 37$ \\
\hline Bulbar weakness & $28(25.9)$ & $18 / 10$ \\
\hline Limb weakness & $44(40.7)$ & $30 / 14$ \\
\hline \multicolumn{3}{|l|}{ Clinical disease type } \\
\hline Ocular MG & $21(19.4)$ & $6 / 15$ \\
\hline Generalized MG & $87(80.6)$ & $55 / 32$ \\
\hline Thymic pathology & 49 & $38 / 11$ \\
\hline Thymoma & $12(24.5)$ & $8 / 4$ \\
\hline Thymus hyperplasia & $28(57.1)$ & $23 / 5$ \\
\hline Normal or atrophic & $9(18.4)$ & $7 / 2$ \\
\hline Concomitant autoimmunity & $22(20.4)$ & $17 / 5$ \\
\hline Graves' disease & $10(9.3)$ & $7 / 3$ \\
\hline Hashimoto thyroiditis & $12(11.2)$ & $10 / 2$ \\
\hline Other & 0 & 0 \\
\hline
\end{tabular}

Values are presented as numbers (\%) unless otherwise stated. MG, myasthenia gravis. ${ }^{a}$ Expressed as mean \pm standard deviation.

to the control subjects, of whom the number who were HLA-B*50 positive was only $7(2.8 \%)$. This difference was still significant after correction for multiple comparisons $(P c=0.00045$, OR 9.92; 95\% CI $=3.05-32.22)$. The clinical features of the HLA-B*50-positive patients (female: male ratio $=3 / 3$ ) were as follows: the mean age at disease onset was $48.5 \pm 23.0$ years, and all patients were seropositive for anti-AchR antibodies; of 6 patients, 2 (33.3\%) had EOMG, 1 (16.7\%) had thymoma, 2 (33.3\%) had thymic hyperplasia, and none had any concomitant autoimmune disease.

\section{Discussion}

In the present study, the frequencies of HLA-B*61 and $C^{*} 05$ were higher in the MG patients than in the controls. HLA-B*61 was serologically grouped with $B^{*} 60$ in the $B^{*} 40$ group. The association of the same locus $\left(B^{*} 40\right)$ was previously pointed out by Fernandez-Mestre et al. [16] in Venezuelan MG patients; however, the significance of the 
Table 2. MHC class I allele distribution in MG patients and controls

\begin{tabular}{|c|c|c|c|c|c|c|c|}
\hline HLA allele & Patients & Controls & $p$ value & HLA allele & Patients & Controls & $p$ value \\
\hline$A^{*} 01$ & $22(20.4)$ & $36(14.4)$ & NS & $B^{*} 27$ & $7(6.5)$ & $15(6.0)$ & NS \\
\hline $\mathrm{A}^{*} 02$ & $47(43.5)$ & $108(43.2)$ & NS & $B^{*} 35$ & $29(26.9)$ & $87(34.8)$ & NS \\
\hline$A^{*} 03$ & 15 (13.9) & $53(21.2)$ & NS & $\mathrm{B}^{*} 37$ & $5(4.6)$ & $4(1.6)$ & NS \\
\hline $\mathrm{A}^{*} 11$ & $17(15.7)$ & $37(14.8)$ & NS & $\mathrm{B}^{*} 38$ & $6(5.6)$ & $18(7.2)$ & NS \\
\hline$A^{*} 23$ & $10(9.3)$ & $15(6.0)$ & NS & $B^{*} 39$ & $4(3.7)$ & $5(2.0)$ & NS \\
\hline $\mathrm{A}^{*} 24$ & $25(23.1)$ & $72(28.8)$ & NS & $B^{*} 41$ & $2(1.9)$ & $11(4.4)$ & NS \\
\hline$A^{*} 25$ & 0 & $3(1.2)$ & NS & $B^{*} 44$ & $16(14.8)$ & $32(12.8)$ & NS \\
\hline$A * 26$ & $7(6.5)$ & $29(11.6)$ & NS & $B^{*} 45$ & 0 & $5(2.0)$ & NS \\
\hline$A^{*} 29$ & $4(3.7)$ & $13(5.2)$ & NS & $B^{*} 47$ & 0 & $1(0.4)$ & NS \\
\hline$A^{*} 30$ & $6(5.6)$ & $14(5.6)$ & NS & $\mathrm{B}^{*} 48$ & 0 & $5(2.0)$ & NS \\
\hline$A^{*} 31$ & $8(7.4)$ & $10(4.0)$ & NS & $B * 49$ & $11(10.2)$ & $26(10.4)$ & NS \\
\hline$A * 32$ & $12(11.1)$ & $27(10.8)$ & NS & $B^{*} 50$ & $7(6.5)$ & $7(2.8)$ & NS \\
\hline$A^{*} 33$ & $5(4.6)$ & $11(4.4)$ & NS & $\mathrm{B}^{*} 51$ & $25(23.1)$ & $56(22.4)$ & NS \\
\hline$A^{*} 68$ & $10(9.3)$ & $16(6.4)$ & NS & $B^{*} 52$ & $9(8.3)$ & $20(8.0)$ & NS \\
\hline$A * 69$ & $1(0.9)$ & $6(2.4)$ & NS & $B^{*} 53$ & 0 & $3(1.2)$ & NS \\
\hline$A^{* 74}$ & 0 & $1(0.4)$ & NS & B*54 & 0 & $3(1.2)$ & NS \\
\hline $\mathrm{Cw}^{*} 01$ & $10(9.3)$ & $43(17.2)$ & NS & $\mathrm{B}^{*} 55$ & $7(6.5)$ & $16(6.4)$ & NS \\
\hline $\mathrm{Cw}^{*} 02$ & $9(8.3)$ & $20(8.0)$ & NS & $B^{*} 56$ & $1(0.9)$ & $4(1.6)$ & NS \\
\hline $\mathrm{Cw}^{*} 03$ & $9(8.3)$ & $25(10.0)$ & NS & $B^{*} 57$ & $6(5.6)$ & $10(4.0)$ & NS \\
\hline $\mathrm{Cw}^{*} 04$ & $31(28.7)$ & $85(34.0)$ & NS & $B^{*} 58$ & $3(2.8)$ & $6(2.4)$ & NS \\
\hline $\mathrm{Cw}^{*} 05$ & $16(14.8)$ & $17(6.8)$ & 0.016 & $B^{*} 60$ & $4(3.7)$ & $10(4.0)$ & NS \\
\hline $\mathrm{Cw}^{*} 06$ & $24(22.2)$ & $44(17.6)$ & NS & $B^{*} 61$ & $8(7.4)$ & $5(2.0)$ & 0.012 \\
\hline $\mathrm{Cw}^{*} 07$ & $35(32.4)$ & $89(35.6)$ & NS & $B^{*} 62$ & $3(2.8)$ & $3(1.2)$ & NS \\
\hline $\mathrm{Cw}^{*} 08$ & 2 (1.9) & $14(5.6)$ & NS & $B^{*} 63$ & 0 & $6(2.4)$ & NS \\
\hline $\mathrm{Cw}^{*} 12$ & $1(0.9)$ & $1(0.4)$ & NS & $B^{*} 64$ & 0 & $2(0.8)$ & NS \\
\hline $\mathrm{Cw}^{*} 17$ & $1(0.9)$ & $5(2.0)$ & NS & $B^{*} 65$ & 0 & $5(2.0)$ & NS \\
\hline $\mathrm{B}^{*} 07$ & $13(12.0)$ & $29(11.6)$ & NS & $\mathrm{B}^{*} 71$ & $1(0.9)$ & 0 & NS \\
\hline $\mathrm{B}^{*} 08$ & $14(13.0)$ & $25(10.0)$ & NS & $\mathrm{B}^{*} 72$ & 0 & $1(0.4)$ & NS \\
\hline $\mathrm{B}^{*} 13$ & $4(3.7)$ & $25(10.0)$ & NS & $\mathrm{B}^{*} 75$ & $2(1.9)$ & $1(0.4)$ & NS \\
\hline $\mathrm{B}^{*} 18$ & $16(14.8)$ & $24(9.6)$ & NS & $B^{*} 87$ & 0 & $1(0.4)$ & NS \\
\hline
\end{tabular}

Values are presented as numbers (\%) unless otherwise stated. MHC, major histocompatibility complex; MG, myasthenia gravis; NS, not significant.

allelic difference was lost after correction of the $p$ value in their study. There were no other allele associations with MG in the present study. Previously, in other studies conducted on Turkish patients, the higher frequencies of HLA-DQB $1 * 05$ (although the $p$ value was not corrected for multiple comparisons) and the 8.1 ancestral haplotype (A1B8DR3 haplotype) had been reported and a significant association with the disease was suggested $[17,18]$. The latter association was also reported by Vandiedonck et al. [19] in French patients with MG of Caucasian origin. On the other hand, MG was associated with HLA-A*25 in Caucasians from France, and with HLA-DQB1*0201 in Swedish MG patients, while HLA-DRB1*09 was positively associated and DRB $1 * 08$ was negatively associated with MG in Northern Han Chinese patients, previously $[13,20,21]$. The reported MG-MHC allele associations seem to differ from population to population, and their contribution to the pathogenesis is still unclear $[14,19$, 22].

The lack of an association with these previously reported HLA alleles could reflect the heterogeneity of the patient population in the present study, together with the contribution of other non-HLA genes and environmental and immunological factors to the pathogenesis. Although, Turkey has a diverse population with multiethnic backgrounds, the control subjects with similar ethnic origins were selected from the Blood and Tissue Bank Registry, correspondingly. Also, despite the small size of the con- 
Table 3. MHC class II allele distribution of the MG patients and controls

\begin{tabular}{|c|c|c|c|}
\hline HLA allele & Patients & Controls & $p$ value \\
\hline $\mathrm{DRB} 1^{*} 01$ & $12(11.1)$ & $20(8.0)$ & NS \\
\hline $\mathrm{DRB} 1 * 03$ & $21(19.4)$ & $40(16.0)$ & NS \\
\hline $\mathrm{DRB}^{*} 04$ & $22(20.4)$ & $76(30.4)$ & NS \\
\hline DRB1*07 & $18(16.7)$ & $41(16.4)$ & NS \\
\hline $\mathrm{DRB} 1 * 08$ & $7(6.5)$ & $12(4.8)$ & NS \\
\hline DRB1*09 & $3(2.8)$ & $6(2.4)$ & NS \\
\hline $\mathrm{DRB}^{*} 10$ & $5(4.6)$ & $14(5.6)$ & NS \\
\hline $\mathrm{DRB}^{*} 11$ & $45(41.7)$ & $81(32.4)$ & NS \\
\hline $\mathrm{DRB}^{*} 12$ & $3(2.8)$ & $8(3.2)$ & NS \\
\hline $\mathrm{DRB}^{*} 13$ & $17(15.7)$ & $57(22.8)$ & NS \\
\hline DRB1*14 & $18(16.7)$ & $25(10.0)$ & NS \\
\hline DRB1*15 & $26(24.1)$ & $55(22.0)$ & NS \\
\hline DRB1*16 & $6(5.6)$ & $26(10.4)$ & NS \\
\hline $\mathrm{DQB} 1 * 02$ & $32(29.6)$ & $59(23.6)$ & NS \\
\hline DQB1*03 & $65(60.2)$ & $171(68.4)$ & NS \\
\hline DQB1*04 & $6(5.6)$ & $11(4.4)$ & NS \\
\hline DQB1*05 & $41(38.0)$ & $93(37.2)$ & NS \\
\hline DQB1*06 & $36(33.3)$ & $76(30.4)$ & NS \\
\hline
\end{tabular}

Values are presented as numbers (\%) unless otherwise stated. MHC, major histocompatibility complex; MG, myasthenia gravis; NS, not significant.

trol group, the HLA frequencies in the control subjects were in accordance with the control groups of previous studies conducted on Turkish populations $[23,24]$.

In the present study, both the patients with ocular weakness at disease onset and those seropositive for antiAchR antibodies had significantly higher frequencies of HLA-B*50 and $C^{*} 05$ alleles than did the controls, but all of these differences lost significance after correction for multiple comparisons. Although, ocular MG has been associated with HLA-DRB1*09 in Chinese patients previously, there was no such association in this study [13].

The major finding of this study is the significantly high frequency of HLA-B*50 in patients with generalized MG with pure ocular disease onset. Moreover, none of the patients with ocular MG had HLA-B*50. In other words, all MG patients who had pure ocular involvement at disease onset and were positive for the HLA-B*50 allele progressed to generalized disease within the first 2 years. Equally important, these patients were all seropositive for anti-AchR antibodies, with no gender predominance. HLA-B*49 and $B^{*} 50$ are serologically classified into the $B * 21$ group. Although the significance of HLA-B*21 or HLA-B*50 is unknown in MG, HLA-B*21 has been as-
Table 4. Significant HLA allele differences in patient subgroups (before Bonferroni's correction)

\begin{tabular}{|c|c|c|c|}
\hline Subgroups/HLA allele & Patients & Controls & $p$ value \\
\hline \multicolumn{4}{|c|}{ Anti-AchR antibodies $(+)$} \\
\hline $\mathrm{B}^{*} 50$ & $7(8.6)$ & $7(2.8)$ & 0.023 \\
\hline $\mathrm{C}^{*} 05$ & $12(14.8)$ & $17(6.8)$ & 0.027 \\
\hline \multicolumn{4}{|l|}{ EOMG } \\
\hline $\mathrm{DRB} 1 * 14$ & $10(20.8)$ & $25(10.0)$ & 0.033 \\
\hline DQB1*02 & $21(43.8)$ & $59(23.6)$ & 0.004 \\
\hline \multicolumn{4}{|l|}{ Very-late onset MG } \\
\hline $\mathrm{B}^{*} 50$ & $3(14.3)$ & $7(2.8)$ & 0.034 \\
\hline $\mathrm{C}^{*} 01$ & 0 & $43(17.2)$ & 0.031 \\
\hline $\mathrm{DQB} 1 * 03$ & $9(42.9)$ & $171(68.4)$ & 0.017 \\
\hline DQB1*06 & $12(57.1)$ & $76(30.4)$ & 0.012 \\
\hline \multicolumn{4}{|l|}{ Ocular MG } \\
\hline$C^{*} 05$ & $4(25.0)$ & $17(6.8)$ & 0.028 \\
\hline $\mathrm{DRB} 1 * 01$ & $4(25.0)$ & $20(8.0)$ & 0.044 \\
\hline DRB1*11 & $11(68.8)$ & $81(32.4)$ & 0.003 \\
\hline \multicolumn{4}{|l|}{ Thymic hyperplasia } \\
\hline$A^{*} 01$ & $9(32.1)$ & $36(14.4)$ & 0.016 \\
\hline$A^{*} 31$ & $4(14.3)$ & $10(4.0)$ & 0.041 \\
\hline $\mathrm{B}^{*} 08$ & $7(25.0)$ & $25(10.0)$ & 0.018 \\
\hline $\mathrm{DRB} 1 * 14$ & $8(28.6)$ & $25(10.0)$ & 0.004 \\
\hline DQB $1 * 03$ & $12(42.9)$ & $171(68.4)$ & 0.007 \\
\hline \multicolumn{4}{|l|}{ Hashimoto thyroiditis } \\
\hline $\mathrm{B}^{*} 39$ & $2(16.7)$ & $5(2.0)$ & 0.036 \\
\hline \multicolumn{4}{|l|}{ Graves' disease } \\
\hline$C^{*} 05$ & $3(30.0)$ & $17(6.8)$ & 0.033 \\
\hline $\mathrm{C}^{*} 07$ & 0 & $89(35.6)$ & 0.017 \\
\hline $\mathrm{DQB} 1 * 03$ & $3(30.0)$ & $171(68.4)$ & 0.017 \\
\hline
\end{tabular}

Values are presented as numbers (\%) unless otherwise stated. HLA, human leucocyte antigen; MG, myasthenia gravis.

sociated with other autoimmune diseases [25]. Verity et al. [26] reported that the TNF-2 polymorphism at the TNF-308 promoter position was associated with HLA$B * 50$, and among HLA-B*50-positive individuals TNF-2 was strongly correlated with ocular Behçet's disease. The significance of this association with ocular involvement is unknown. Interestingly, in another study, the TNF-308 allele 2 polymorphism was associated with MG, which is associated with higher inducible levels of TNF- $\alpha$ [27]. Although the exact mechanism underlying this genetic association is unknown, the observed high frequency of HLA- $B^{*} 50$ in patients with generalized MG with pure ocular onset might suggest a contributory role for high TNF production and dysregulation of the immune system.

In the present study, although the differences lost significance after correction for multiple comparisons, it 
was found that the HLA-A*01, HLA-A*31, HLA-B*08, HLA-DRB1* 14 , and HLA-DQB1*02 alleles were more frequent in EOMG. On the other hand, there was no significant allele association with LOMG, whereas LOMG has been associated with DRB1*15 in Norwegian patients previously [14]. The observed association between the $\mathrm{DQB} 1 * 02$ allele and EOMG confirmed the results of one of the previous studies conducted on Turkish MG patients [17]. In the other study of Turkish patients with $\mathrm{MG}$, the higher frequencies of the HLA alleles $\mathrm{A}^{*} 02, \mathrm{~B}^{*} 8$, and $\mathrm{DRB} 1 * 03$ were associated with EOMG [18].

The patients with thymic hyperplasia had significantly higher frequencies of HLA-A*31, HLA-B*08, and HLADRB1*14 compared to the controls, though not after correction of $p$ values. This finding supports the results of Fernandez-Mestre et al. [16], as the frequencies of HLA$A * 31$ and HLA-B*08 were also higher in their Venezuelan patients with thymic hyperplasia. In contrast, we found no significant differences in allele frequencies between the patients with thymoma and the controls. The role of the thymus is distinctive in MG, and its abnormality plays an essential role in autoantibody production and autoimmunity. Peptides displayed by antigen-presenting cells via MHC class II alleles in the thymus shape the Tcell repertoire [28]. Thymic pathology has been associated with several HLA alleles. An HLA-A*24 association with thymoma and the ancestral suprahaplotype A1 B8 DRB1*0301 DRB3*0101 DQA1*0501 in association with thymic hyperplasia was reported in 12 German patients [29]. A negative association of HLA-A*02 with thymoma was found in Caucasians from France [20]. Also, 2 studies reported associations between HLA-DRB1*11 in Mexicans and DQ6 in Chinese patients with thymoma $[12,30]$.
This cohort consisted mainly of adult patients, with the exception of 5 adolescence-onset cases. Since the childhood MG has different characteristics in clinical aspects, it can be postulated that different genetic predispositions might affect children. Thus, these findings should not be generalized to the entire MG disease spectrum.

This study had several limitations. First, a case of a selection bias in the controls could arise from the religions of the organ donors (as an effect of ethnicity), but it should be stated that all participants in this study had the same religion. Second, the lack of data on the presence of anti-MuSK antibodies in anti-AChR negative patients was a limitation; this data could add substantial information regarding HLA allele associations with anti-MuSK antibody positivity.

\section{Conclusion}

The most prominent finding of this study is the significantly high frequency of the HLA-B*50 allele in patients with generalized $M G$ who had pure ocular symptoms at disease onset. HLA-B*50 allele positivity was also associated with conversion to generalized disease in patients with pure ocular involvement at disease onset. This finding could substantially add to the understanding of the role of the $\mathrm{MHC}$ region in this disease.

\section{Disclosure Statement}

None.

\section{References}

1 Vincent A, Palace J, Hilton-Jones D: Myasthenia gravis. Lancet 2001;357:2122-2128.

2 Giraud M, Vandiedonck C, Garchon HJ: Genetic factors in autoimmune myasthenia gravis. Ann NY Acad Sci 2008;1132:180-192.

3 Thorsby E, Lie BA: HLA associated genetic predisposition to autoimmune diseases: genes involved and possible mechanisms. Transpl Immunol 2005; 14:175-182.

4 Szobor A: Myasthenia gravis: familial occurrence - a study of 1,100 myasthenia gravis patients. Acta Med Hung 1989;46:13-21.

5 Zhu WH, Lu JH, Lin J, et al: HLA-DQA1*03: 02/DQB1*03:03:02 is strongly associated with susceptibility to childhood-onset ocular myasthenia gravis in Southern Han Chinese. J Neuroimmunol 2012;247:81-85.
6 Protti MP, Manfredi AA, Straub C, et al: $\mathrm{CD} 4+\mathrm{T}$ cell response to the human acetylcholine receptor alpha subunit in myasthenia gravis: a study with synthetic peptides. J Immunol 1990;144:1276-1281.

7 Oshima M, Deitiker PR, Smith RG, et al: $\mathrm{T}$-cell recognition of acetylcholine receptor provides a reliable means for monitoring autoimmunity to acetylcholine receptor in antibody-negative myasthenia gravis patients. Autoimmunity 2012;45:153-160.

8 Vincent A: Unravelling the pathogenesis of myasthenia gravis. Nat Rev Immunol 2002;2: 797-804.

9 Wittbrodt ET: Drugs and myasthenia gravis: an update. Arch Intern Med 1997;157:399408.
10 von Herrath MG, Fujinami RS, Whitton JL: Microorganisms and autoimmunity: making the barren field fertile? Nat Rev Microbiol 2003;1:151-157.

11 Pekiner FN, Aytugar E, Demirel GY, et al: HLA-A, B (class I) and HLA-DR, DQ (class II) antigens in Turkish patients with recurrent aphthous ulceration and Behcet's disease. Med Princ Pract 2013;22:464-468.

12 Garcia-Ramos G, Tellez-Zenteno JF, ZapataZuniga $\mathrm{M}$, et al: HLA class II genotypes in Mexican mestizo patients with myasthenia gravis. Eur J Neurol 2003;10:707-710.

13 Xie YC, Qu Y, Sun L, et al: Association between HLA-DRB1 and myasthenia gravis in a Northern Han Chinese population. J Clin Neurosci 2011;18:1524-1527. 
14 Maniaol AH, Elsais A, Lorentzen AR, et al: Late onset myasthenia gravis is associated with HLA DRB1*15:01 in the Norwegian population. PLoS One 2012;7:e36603.

15 Fekih-Mrissa N, Klai S, Zaouali J, et al: Association of HLA-DR/DQ polymorphism with myasthenia gravis in Tunisian patients. Clin Neurol Neurosurg 2013;115:32-36.

16 Fernandez-Mestre MT, Vargas V, Montagnani S, et al: HLA class II and class I polymorphism in Venezuelan patients with myasthenia gravis. Hum Immunol 2004;65:54-59.

17 Saruhan-Direskeneli G, Kilic A, Parman Y, et al: HLA-DQ polymorphism in Turkish patients with myasthenia gravis. Hum Immunol 2006;67:352-358.

18 Donmez B, Ozakbas S, Oktem MA, et al: HLA genotypes in Turkish patients with myasthenia gravis: comparison with multiple sclerosis patients on the basis of clinical subtypes and demographic features. Hum Immunol 2004; 65:752-757.

19 Vandiedonck C, Beaurain G, Giraud M, et al: Pleiotropic effects of the 8.1 HLA haplotype in patients with autoimmune myasthenia gravis and thymus hyperplasia. Proc Natl Acad Sci USA 2004;101:15464-15469.
20 Vandiedonck C, Raffoux C, Eymard B, et al: Association of HLA-A in autoimmune myasthenia gravis with thymoma. J Neuroimmunol 2009;210:120-123.

21 Hjelmstrom P, Giscombe R, Lefvert AK, et al: Different HLA-DQ are positively and negatively associated in Swedish patients with myasthenia gravis. Autoimmunity 1995;22:5965.

22 Deitiker PR, Oshima M, Smith RG, et al: Association with HLA DQ of early onset myasthenia gravis in Southeast Texas region of the United States. Int J Immunogenet 2011;38: 55-62.

23 Aydogan K, Karadogan SK, Akdag I, et al: HLA class I and class II antigens in Turkish patients with chronic ordinary urticaria. Clin Exp Dermatol 2006;31:424-429.

24 Bilir M, Sipahi S, Yilmaz E, et al: Analysis of HLA antigens in Turkish sarcoidosis patients. South Med J 2007;100:356-359.
25 Nanda A, Alsaleh QA, Al-Hasawi F, et al: Thyroid function, autoantibodies, and HLA tissue typing in children with alopecia areata. Pediatr Dermatol 2002;19:486-491.

26 Verity DH, Wallace GR, Vaughan RW, et al: HLA and tumour necrosis factor (TNF) polymorphisms in ocular Behcet's disease. Tissue Antigens 1999;54:264-272.

27 Huang DR, Pirskanen R, Matell G, et al: Tumour necrosis factor-alpha polymorphism and secretion in myasthenia gravis. J Neuroimmunol 1999;94:165-171.

28 Melms A, Luther C, Stoeckle C, et al: Thymus and myasthenia gravis: antigen processing in the human thymus and the consequences for the generation of autoreactive T cells. Acta Neurol Scand Suppl 2006;183:12-13.

29 Machens A, Loliger C, Pichlmeier U, et al: Correlation of thymic pathology with HLA in myasthenia gravis. Clin Immunol 1999;91: 296-301.

30 Yang $\mathrm{H}$, Hao J, Peng $\mathrm{X}$, et al: The association of HLA-DQA $1 * 0401$ and DQB1*0604 with thymomatous myasthenia gravis in Northern Chinese patients. J Neurol Sci 2012;312:5761. 